\title{
Drell-Yan as an avenue to test noncommutative Standard Model at the Large Hadron Collider
}

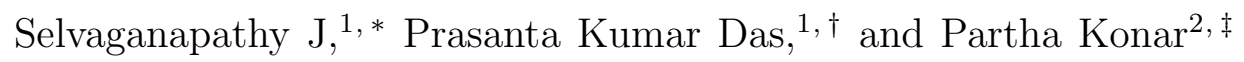 \\ ${ }^{1}$ Department of Physics, Birla Institute of Technology and Science-Pilani, \\ K K Birla Goa campus, Goa-403726, India \\ ${ }^{2}$ Physical Research Laboratory, Ahmedabad-380009, India
}

\begin{abstract}
We study the Drell-Yan process at the Large Hadron Collider in presence of the noncommutative extension of standard model. Using the Seiberg-Witten map, we calculate the production cross-section to the first order in the noncommutative parameter $\Theta_{\mu \nu}$. Although this idea is evolving for long, limited amount of phenomenological analysis was done so far and dominantly in the context of linear collider. Some outstanding feature from this non-minimal noncommutative standard model not only modify the couplings over SM production channel, also allow additional nonstandard vertices which can play a significant role. Hence in the Drell-Yan process, as studied in present analysis, one also needs to account for gluon fusion process at the tree level. Some of the characteristic signatures such as oscillatory azimuthal distributions are outcome of the momentum dependent effective couplings. We explore the noncommutative scale $\Lambda_{N C} \geq 0.4 \mathrm{TeV}$ considering different machine energy ranging from $7 \mathrm{TeV}$ to $13 \mathrm{TeV}$.

PACS numbers: $11.10 . \mathrm{Nx}, 12.60 .-\mathrm{i}, 14.80 . \mathrm{Ec}$
\end{abstract}

Keywords: Non-Commutative Standard Model, Hadronic Colliders, Drell-Yan process

*Electronic address: p2012015@goa.bits-pilani.ac.in

$\dagger$ Electronic address: pdas@goa.bits-pilani.ac.in

${ }^{\ddagger}$ Electronic address: konar@prl.res.in 


\section{INTRODUCTION}

The Large Hadron Collider (LHC) has so far been extremely successful in discovering and constraining the properties of the last missing bit of the Standard Model (SM) of particle physics, the Higgs boson [1, 2]. Apart from some isolated hints, it is broadly evasive lacking any clinching evidence yet from the physics beyond the Standard Model (BSM), exploration of which is one of the primary motive for post-Higgs LHC. On the other side, it is widely admitted that the SM can at most a very good description for low energy effective theory which, in fact, falls short to explain several outstanding issues both in theoretical expectations, as well as experimental observations.

The idea of field theories on the noncommutative (NC) spacetime is rather primeval, yet fascinating by introducing a fundamental length scale in the model consistent with the symmetry [3]. These ideas are further revived after realisation of their possible connection with the quantum gravity, where noncommutativity is perceived as an outcome of certain string theory embedded into a background magnetic field 44. Quantum field theory is described by the fields and the local interaction in a continuous space-time point, where the canonical position and momentum variables $x_{i}, p_{j}$ are replaced by the operator $\hat{x}_{i}, \hat{p}_{j}$ which satisfy the commutation relation

$$
\left[\hat{x}_{i}, \hat{p}_{j}\right]=i \hbar \delta_{i j}
$$

Just like the quantisation in phase space, the spacetime coordinate in the noncommutative spacetime gets replaced by an operator $\hat{x}_{\mu}$ which satisfy the commutation relation

$$
\left[\hat{x}_{\mu}, \hat{x}_{\nu}\right]=i \Theta_{\mu \nu}=i \frac{c_{\mu \nu}}{\Lambda_{N C}^{2}}
$$

where $\Theta_{\mu \nu}$ is an antisymmetric matrix tensor and of dimension $[M]^{-2}$. Now, one can take out the dimension full part in terms of mass parameter $\Lambda_{N C}$ and describe it as the fundamental NC scale at which one expects to see the effect of spacetime noncommutativity. $c_{\mu \nu}$ is the anti-symmetric constant c-number matrix which gives a preferred directionality and also a non-vanishing contribution results in deviating from exact Lorentz invariance in some high energy scale $\Lambda_{N C}$. Theoretically this scale is unknown, but one can try to extract the lower bounds directly from the collider experiments by looking at the characteristic signals this framework can provide. LEP studied the prediction of the process $e^{+} e^{-} \rightarrow \gamma \gamma$ in the noncommutative QED for several orientation of the OPAL detector and provided 
the exclusion limit at around $141 \mathrm{GeV}$ [5]. With this very moderate bound, we have scope for significant improvement at the future runs of LHC. Drell-Yan process is arguably best explored process at hadron collider. With an extremely clean signals of di-lepton, this process is relied upon for calibrating the parton distribution function in hadrons. Any characteristic deviation can easily be a basis for the BSM search. In our present work, we study this important process in the context of noncommutative framework.

There are different approaches to study the effect of spacetime noncommutativity in a field theory. One is the Moyal-Weyl (MW) approach. In this approach, one replaces the ordinary product between two functions $\phi(x)$ and $\psi(x)$ in terms of $\star$ (Moyal-Weyl) product defined by a formal power series expansion of $6-8$

$$
(f \star g)(x)=\left.\exp \left(\frac{1}{2} \Theta_{\mu \nu} \partial_{x^{\mu}} \partial_{y^{\nu}}\right) f(x) g(y)\right|_{y=x} .
$$

Here $f(x)$ and $g(x)$ are ordinary functions on $R^{n}$ and the expansion in the star product can be seen intuitively as an expansion of the product in its non-commutativity. To describe some of the collider searches of spacetime noncommutativity available in the literature, Hewett et al. [9, 10] have studied the processes $e^{+} e^{-} \rightarrow e^{+} e^{-}$(Bhabha) and $e^{-} e^{-} \rightarrow e^{-} e^{-}$(Moller) and subsequent studies [11, 12] were done in the context of $e \gamma \rightarrow e \gamma$ (Compton) and $e^{+} e^{-} \rightarrow \gamma \gamma$ (pair annihilation), $\gamma \gamma \rightarrow e^{+} e^{-}$and $\gamma \gamma \rightarrow \gamma \gamma$. For a review on NC phenomenology, see [22]. In the context of LHC, the following investigations are in order. Noncommutative contribution of neutral vector boson $(\gamma, z)$ pair production was studied [23] at the LHC and obtained the bound for the NC scale $\Lambda \geq 1 \mathrm{TeV}$ under some conservative assumptions. Further study on the pair producton of charged gauge bosons $\left(W^{ \pm}\right)$at the LHC in the noncommutative extension of the standard model found [24] significant deviation of the azimuthal distribution(oscillation) from the SM one (which is a flat distribution) for $\Lambda_{N C}=$ $700 \mathrm{GeV}$. More recently, t-channel single top quark production is calculated at the LHC and found significant deviation in cross section can be expected from the Standard Model for $\Lambda_{N C} \geq 980 \mathrm{GeV}[25]$.

Second way of dealing this calculation is the Seiberg-Witten approach in which the spacetime noncommutativity is being treated perturbatively via the Seiberg-Witten (SW) map expansion of the fields in terms of noncommutative parameter $\Theta$ [4]. Here the gauge parameter $\lambda$ and the gauge field $A^{\mu}$ is expanded as

$$
\lambda_{\alpha}(x, \Theta)=\alpha(x)+\Theta^{\mu \nu} \lambda_{\mu \nu}^{(1)}(x ; \alpha)+\Theta^{\mu \nu} \Theta^{\eta \sigma} \lambda_{\mu \nu \eta \sigma}^{(2)}(x ; \alpha)+\cdots
$$




$$
A_{\rho}(x, \Theta)=A_{\rho}(x)+\Theta^{\mu \nu} A_{\mu \nu \rho}^{(1)}(x)+\Theta^{\mu \nu} \Theta^{\eta \sigma} A_{\mu \nu \eta \sigma \rho}^{(2)}(x)+\cdots
$$

The advantage in the SW aprroach over the Weyl-Moyal approach is that it can be applied to any gauge theory and matter can be in an arbitrary representation. Using this SW map Calmet et al. first constructed [13, 14] the minimal version of the noncommutative standard model (mNCSM in brief) where they derived the $\mathcal{O}(\Theta)$ Feynman rules of the standard model interactions and found several new interactions which are not present in the standard model. All the above analyses were limited to the leading order in $\Theta$. Phenomenological analysis was carried out for the process $e^{+} e^{-} \rightarrow \gamma, Z \rightarrow \mu^{+} \mu^{-}$at the order of $\Theta^{2}$ and predicted a reach of around $\Lambda_{N C}=800 \mathrm{GeV}$ for the $\mathrm{NC}$ scale [15, 16]. successive study [17] also focused on the top quark pair production in the NCSM and predicted a similar reach of the NCSM scale.

The non-commutative standard model essentially the standard model with the background space-time being non-commutative. Contrary to most other BSM models where particle content and/or the gauge group is extended, there is no new massive degree of freedom is included here, but the standard model interactions get modified due to space-time noncommutativity. This gives rise the modified standard model interaction vertices extending with additional $\mathrm{NC}$ contributions. Moreover, it also provides a host of new vertices which are absent in the SM. It is demonstrated [13, 14, 18, that the minimal version of NCSM can only be realised in some definite choice of representation for the traces in gauge field kinetic term. Freedom of this choice leads to a more natural extended version. Melic et al. [20, 21] formulated the non-minimal version of NCSM (nmNCSM in brief), where the trilinear neutral gauge Boson couplings arise automatically. Note that such anomalous vertices were absent in the minimal version and the interactions in fermion sector remain unaffected by different choices of representation in gauge action. Using this formalism associated Higgs Boson production is recently studied associated with the $Z$ boson, taking into account of the earth rotation effect in the nmNCSM and found that the azimuthal distribution significant differs from the standard model result if the NC scale $\Lambda_{N C} \geq 500 \mathrm{GeV}$ [19].

Exotic new vertices can serve as the Occam's Razor in differentiating the NCSM from other new physics models. In this paper, we discuss this possibility considering one of the very simple but reliable signature at the hadron collider which can portray its ability by distinguishing the effects of space-time noncommutativity from other new physics scenarios. 
Our analysis is based on the parton level Drell-Yan process in producing the lepton pair at the large hadron collider

$$
p p \rightarrow l^{+} l^{-}+X
$$

where light leptons $(l \equiv e, \mu)$ of opposite sign is produced at the final state. What is significant here, besides the standard quark initiated partonic sub-process for di-lepton production, gluon initiated processes can also contribute to this production cross-section. In the second process new triple gauge boson vertices $K_{Z g g}$ and $K_{\gamma g g}$ contribute which arise naturally as an effective vertex in noncommutativity although forbidden in the Standard Model. A study of these vertices have been performed by Behr et al. [18]. Using the experimental LEP upper bound from $\Gamma_{Z \rightarrow \gamma \gamma}^{e x p}<1.3 \times 10^{-4} \mathrm{GeV}$ and $\Gamma_{Z \rightarrow g g}^{\exp }<1.0 \times 10^{-3} \mathrm{GeV}$, a correlated bound on these vertices were obtained for $\mathrm{NC}$ scale $\Lambda_{N C}=1 \mathrm{TeV}^{1}$.

The organization of the paper is as follows. In Sec. II] we describe the modified vertices and the new set of vertices (found to be absent in the SM) which may potentially contribute to the Drell-Yan lepton pair production at the hadron collider. We next obtain the matrix element square for the partonic sub-process and obtain the total cross-section, differential cross-section of Drell-Yan lepton pair production. In Sec. III, we demonstrate some of the characteristic distributions, such as, lepton pair invariant mass distribution, total cross-section, angular distribution. Arguing in favour of them carrying the hallmark for noncommutative effects we probe the sensitivity of the new vertices to the Drell-Yan lepton pair production. Finally, in Sec. IV] we summarize our result and conclude.

\section{DRELL-YAN PRODUCTION IN NONCOMMUTATIVE STANDARD MODEL}

At the LHC lepton pairs can be produced at the tree-level via the (quark-like) parton initiated process (the only partonic level processes possible in the SM at the tree level)

$$
q \bar{q} \rightarrow \gamma, Z \rightarrow l^{+} l^{-}
$$

\footnotetext{
${ }^{1}$ Note that, in principle, $K_{Z g g}$ (and $K_{\gamma g g}$ ) can be zero, in combination of other two couplings $K_{\gamma \gamma \gamma}$ and $K_{Z \gamma \gamma}$. But all three cannot be zero [18] simultaneously and these other two couplings can be tested at the linear collider with high degree of precession. We discuss this allowed range in the next section, and also demonstrate our results considering different values within this range.
} 


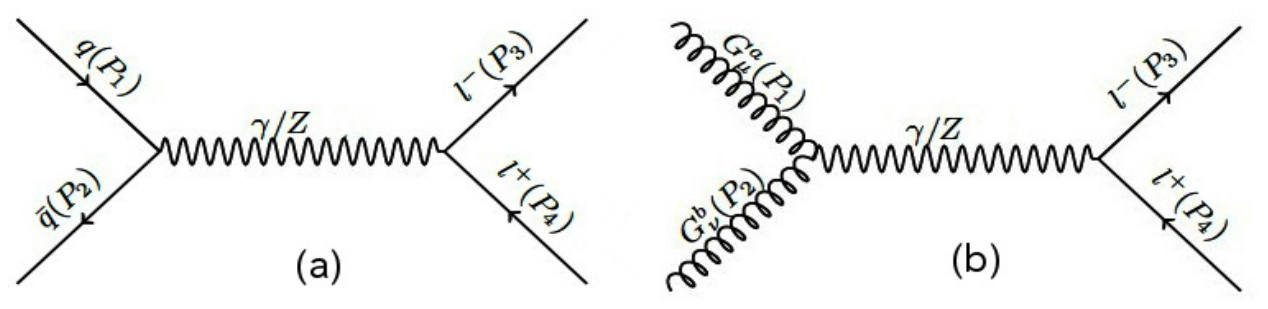

FIG. 1: Representative Feynman diagrams for the partonic subprocess for quark initiated (a) $q \bar{q} \rightarrow \gamma, Z \rightarrow l^{+} l^{-}$, and gluon initiated (b) $g g \rightarrow \gamma, Z \rightarrow l^{+} l^{-}$. Both of them contributes in DrellYan type lepton pair production at the hadron collider considering noncommutative standard model.

together with, in the NCSM additional three boson vertices ensures that lepton pairs can also be produced at the tree-level through gluon fusion,

$$
g g \rightarrow \gamma, Z \rightarrow l^{+} l^{-}
$$

Representative Feynman diagrams for these partonic subprocess are shown in Fig. 1. For the quark mediated process, the Feynman rules for the vertices $f \bar{f} \gamma$ and $f \bar{f} Z$ (where $f=q, l$ ) are shown in Appendix A. Note that the vertices, besides the SM part, also contain extra $\mathcal{O}\left(\Lambda_{N C}\right)$ dependent term for which, at the limit $\Lambda_{N C} \rightarrow \infty$, the original SM vertices get recovered. The second gluon mediated partonic-process comprises two new vertices $\gamma g g$ and $Z g g$, which are not present in the SM and depicted in Fig. 2. Corresponding leading order
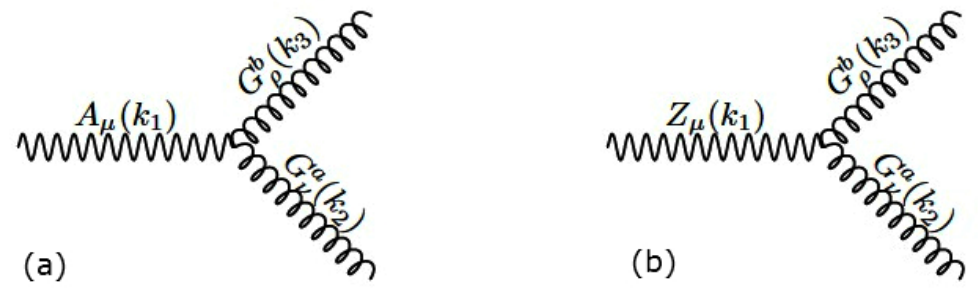

FIG. 2: Feynman diagrams for additional vertices in the noncommutative standard model which can contribute in Drell-Yan production process at the LHC.

Feynman rules in these figures are given by,

$$
\begin{aligned}
\gamma g g & :(-2 e) \sin \left(2 \theta_{w}\right) K_{\gamma g g} \theta_{3}^{\mu \nu \rho}\left(k_{1}, k_{2}, k_{3}\right) \delta^{a b} \\
Z g g & :(-2 e) \sin \left(2 \theta_{w}\right) K_{Z g g} \theta_{3}^{\mu \nu \rho}\left(k_{1}, k_{2}, k_{3}\right) \delta^{a b} .
\end{aligned}
$$


Here, $\theta_{W}$ is the Weinberg angle and the vertex factors $K_{\gamma g g}$ and $K_{Z g g}$ are given by

$$
K_{\gamma g g}=\frac{-g_{s}^{2}}{2 g g^{\prime}}\left(g^{\prime 2}+g^{2}\right) \zeta_{3}, \quad K_{Z g g}=\left(-\tan \theta_{w}\right) K_{\gamma g g},
$$

where $g_{s}, g, g^{\prime}$ are being the $S U(3)_{C}, S U(2)_{L}$ and $U(1)_{Y}$ coupling strengths, respectively. The tensorial quantity ${ }^{2} \theta_{3} \equiv \theta_{3}^{\mu \nu \rho}\left(k_{1}, k_{2}, k_{3}\right)$ and the parameter $\zeta_{3}$ are defined in Appendix A. Note that the triple gauge boson vertices $K_{Z g g}$ and $K_{\gamma g g}$, absent in the Standard Model (once again, one gets a vanishing $\theta_{3}$ at the limit $\Lambda_{N C} \rightarrow \infty$ ), arise in this nonminimal version of NCSM. A direct test of these vertices have been performed [18] by studying the $\mathrm{SM}$ forbidden decays $Z \rightarrow \gamma \gamma$ and $Z \rightarrow g g$. Analyzing the 3-dimensional simplex that bounds possible values for the coupling constants $K_{\gamma \gamma \gamma}, K_{Z \gamma \gamma}$ and $K_{Z g g}$ at the $M_{Z}$ scale,

allowed region for our necessary couplings $\left(K_{Z g g}, K_{Z \gamma \gamma}\right)$ are obtained as ranging between $(-0.108,-0.340)$ and $(0.217,-0.254)$.

\section{RESULT AND DISCUSSION}

To estimate the noncommutative effects in our parton level calculation, we analytically formulate both subprocess initiated either by quark-antiquark pair or by gluon pair at the leading order. Using the Feynman rules to $\mathcal{O}(\Theta)$ as described above and in Appendix A, the squared amplitude (spin-averaged) can be expressed as,

$$
{\overline{\left|M_{N C S M}^{2}\right|}}_{a b \rightarrow l^{+} l^{-}}=\overline{\left|\mathcal{M}_{\gamma}+\mathcal{M}_{Z}\right|^{2}} \quad \text { for, } a, b=q, \bar{q} \text { or } g, g \text {. }
$$

Detailed analytic expression for each nmNCSM amplitude-square is presented in Appendix B. The $\mathrm{NC}$ antisymmetric tensor $\Theta_{\mu \nu}$, analogous to the electro-magnetic field(photon) strength tensor, has 6 independent components: 3 are of electric type, while 3 are of magnetic type. We have chosen $E_{i}=\frac{1}{\sqrt{3}}$ and $B_{i}=\frac{1}{\sqrt{3}}$ in our analysis. (for more, see the Appendix C). We have not considered here the effect of earth rotation. The impact of earth rotation on $\Theta_{\mu \nu}$ in DY process can be interesting, which is investigated in a future work. [26]. Also note that in the DY lepton distribution, besides the Lorentz invariant momentum dot product (e.g. $p_{1} \cdot p_{2}$ etc), the $\Theta$-wieghted dot product (e.g. $p_{3} \Theta p_{4}$ as one follows from Appendix C) also appears. These terms give rise non-trivial azimuthal distribution in the Drell-Yan lepton pair production as discussed at the end of our results.

\footnotetext{
${ }^{2}$ We follow the couplings in similar notation as in 18
} 

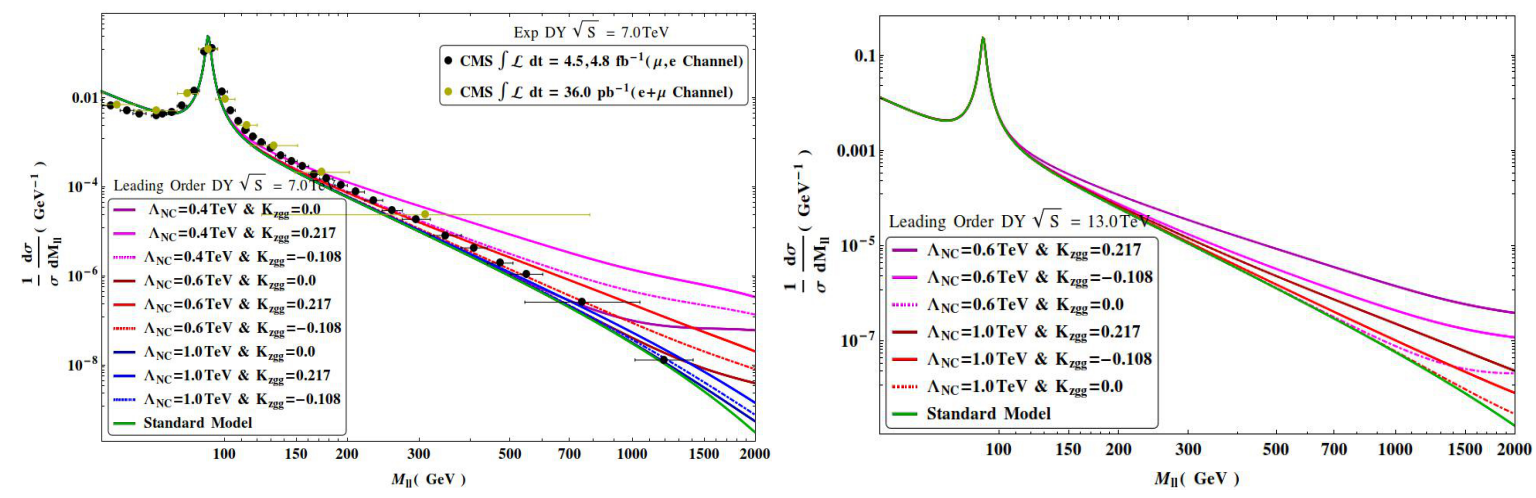

FIG. 3: (Color online). Normalized invariant mass distribution $\frac{1}{\sigma} \frac{d \sigma}{d M_{l l}}\left(\mathrm{GeV}^{-1}\right)$ as a function of the invariant mass $M_{l l}(\mathrm{GeV})$ is shown corresponding to the machine energy (left plot) $\sqrt{s}=7 \mathrm{TeV}$ and (right plot) $13 \mathrm{TeV}$ respectively. Continuous curves of different colours in both plots are shown for the choice of $\Lambda$ and $K_{Z g g}$ and they converge to the lowermost SM curve in the limit both of these parameters go to zero. In $7 \mathrm{TeV}$ plot, experimental bin-wise data is also shown with central values and error bars.

We estimate the parton level total cross section and differential distributions for the LHC operated at the energy $\sqrt{S}$,

$$
d \sigma_{p p \rightarrow l^{+} l^{-}}=\sum_{a b} \int d x_{1} \int d x_{2} f_{a}\left(x_{1}, \mu_{f}^{2}\right) f_{b}\left(x_{2}, \mu_{f}^{2}\right) d \hat{\sigma}_{a b \rightarrow l^{+} l^{-}}\left(x_{1} x_{2} S\right) .
$$

We employ CTEQ6L1 parton distribution function (PDF) throughout the analysis, setting the factorization scale $\mu_{f}$ at the dilepton invariant mass $M_{l l}$. After formulating the setup, we are now in a position to describe the numerical results for the Drell-Yan lepton pair production in the presence of spacetime noncommutativity. In Fig. 3 we have shown the normalized di-lepton invariant mass distribution $\frac{1}{\sigma} \frac{d \sigma}{d M_{l l}}\left(\mathrm{GeV}^{-1}\right)$ against the invariant mass $M_{l l}(\mathrm{GeV})$ corresponding to the LHC machine energy $\sqrt{s}$ at (left plot) $7 \mathrm{TeV}$ and (right plot) $13 \mathrm{TeV}$. The peak at $M_{l l}=91.18 \mathrm{GeV}$ corresponds to the $Z$ boson resonance production. Different continuous curves in both plots correspond to the theoretical (SM and nmNCSM) predictions. Note that the additional positive contributions in nmNCSM curves are realised from two sources; first being the $\Theta$ dependent NC parts supplemented with the SM vertex, and the second being complete new tree level process that enhances significantly. In the $7 \mathrm{TeV}$ (left) plot the dotted curves correspond to the experimental binwise data provided by CMS collaboration [27] for the integrated luminosity $4.5 \mathrm{fb}^{-1}$ and $35.9 \mathrm{pb}^{-1}$ which is 


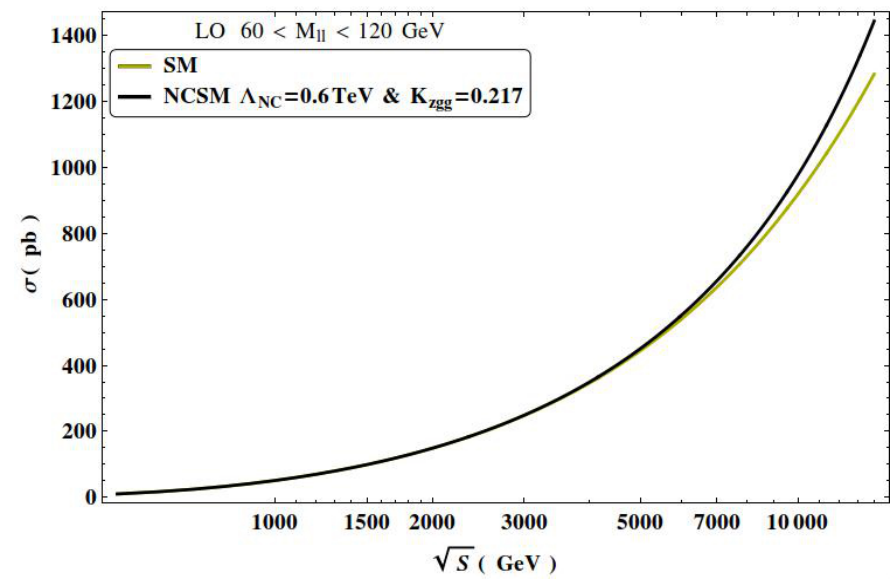

FIG. 4: (Color online). The Drell-Yan cross section is shown as a function of the LHC machine energy. In the NCSM, we demonstrate with one of the very optimistic choice like, $\Lambda_{N C}=0.6 \mathrm{TeV}$ and $K_{Z g g}=0.217$.

presented along with the error bar.

The lowermost curve in each plot, Fig. 3 is the SM contribution estimated at the leading order. In this figure we present different NC contribution based on the two relevant parameters $\Lambda_{N C}$ and $K_{Z g g}$ varying between $(0.4 \mathrm{TeV}-1 \mathrm{TeV})$ and $(-0.108,+0.217)$ respectively. Justification for these choices are already discussed before. Since the parameter $K_{Z g g}$ contributes in square from gluon initiated diagram, sign of this parameter is irrelevant. So, both sign contribute positively and the magnitude depending upon the absolute values. Note that $K_{Z g g}=0$ corresponds to the vanishing coupling of gluon with $Z$ and $\gamma$ bosons and and the Drell-Yan process in the NCSM arises only from the quark mediated partonic subprocess $q \bar{q} \rightarrow \gamma, Z \rightarrow l^{+} l^{-}$as in Figure 1(a). The NC scale $\Lambda_{N C}$ determines the energy when this BSM effects can be perceived and this phenomena is evident following different scales in the figure. At around few hundred of dilepton invariant mass Fig. 3 exibits, especially at the low $\Lambda_{N C}$ and larger absolute value of $K_{Z g g}$, the NCSM effect in this distribution deviating from the SM distribution and it increases with $M_{l l}$.

In Fig. 4, we have plotted total leading order Drell-Yan cross section $\sigma$ (in pb) against the LHC collision energy $\sqrt{s}$. The lower curve corresponds to the SM cross section. We find $\sigma=635(1283) \mathrm{pb}$ at $\sqrt{s}=7(14) \mathrm{TeV}$, respectively. To estimate the total cross section, we have considered the di-lepton invariant mass interval $60 \mathrm{GeV}<\mathrm{M}_{11}<120 \mathrm{GeV}$. To visualise the effect we once again consider a very optimistic values of $\Lambda_{N C}=0.6 \mathrm{TeV}$ and 


\begin{tabular}{|c|c|c|c|}
\hline$\sqrt{S} \mathrm{TeV}$ & $\sigma_{S M}(p b)$ & $\sigma_{n m N C S M}(p b)$ & $\sigma_{E X P}(p b)$ \\
& $\mathrm{LO}, \mu_{f}=M_{z}$ & $\mathrm{LO}, \mu_{f}=M_{z}$ & \\
\hline 7.0 & 636 & 656 & $974 \pm 0.7$ (Stat) \pm 0.7 (Syst) \\
8.0 & 731 & 760 & $1138 \pm 8$ (Stat) \\
13.0 & 1193 & 1325 & \\
14.0 & 1283 & 1438 & \\
\hline
\end{tabular}

TABLE I: Drell-Yan cross section in the SM, nmNCSM are shown for $60 \mathrm{GeV}<\mathrm{M}_{11}<120 \mathrm{GeV}$. The experimental data for the same di-lepton invariant mass interval is shown. Here we have set the parameters $\Lambda_{N C}=0.6 \mathrm{TeV}$ and $K_{Z g g}=0.217$ which is optimistic.

$K_{Z g g}=0.217$ for the upper curve corresponds to the NCSM cross section. For reference we present the corresponding Drell-Yan cross sections for different machine energy in Table I. For different machine energy between $7 \mathrm{TeV}$ and $14 \mathrm{TeV}$, the leading order $\mathrm{SM}$ and the nmNCSM (for the same reference parameters) cross sections increases from 636 (656)pb to $1283(1438) \mathrm{pb}$.

In Fig. 5, the nmNCSM Drell-Yan cross section is shown as a function of the NC scale $\Lambda_{N C}$ at a fixed machine energy $\sqrt{s}=7.0 \mathrm{TeV}$. Once again dominant production cross section is estimated for the range of invariant mass $60 \mathrm{GeV}<\mathrm{M}_{11}<120 \mathrm{GeV}$ corresponding to different values of the parameter $K_{Z g g}=-0.108,0.0,0.0545$ and 0.217 . As expected, for a fixed $K_{Z g g}$

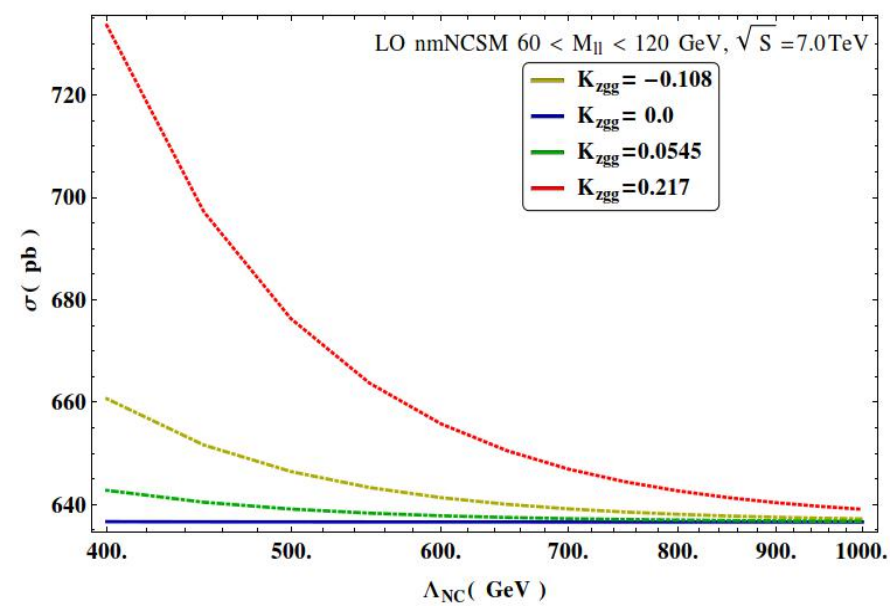

FIG. 5: The total cross section for $p p \rightarrow(\gamma, Z) \rightarrow l^{+} l^{-} \sigma$ is plotted as a function of the NC scale $\Lambda_{N C}(\mathrm{GeV})$ corresponding to $Z=-0.108,0.054$ and 0.217 and fixed machine energy $\sqrt{s}=7.0 \mathrm{TeV}$. 


\begin{tabular}{|c|c|c|c|c|c|c|c|c|}
\hline$\Lambda_{N C}(\mathrm{TeV})$ & $K_{Z g g}$ & $\sigma_{N C S M}(\mathrm{pb})$ & $\Lambda_{N C}(\mathrm{TeV})$ & $K_{Z g g}$ & $\sigma_{N C S M}(\mathrm{pb})$ & $\Lambda_{N C}(\mathrm{TeV})$ & $K_{Z g g}$ & $\sigma_{N C S M}(\mathrm{pb})$ \\
\hline 0.4 & 0.0 & 637 & 0.6 & 0.0 & 637 & 1.0 & 0.0 & 637 \\
0.4 & -0.108 & 661 & 0.6 & -0.108 & 641 & 1.0 & -0.108 & 637 \\
0.4 & 0.217 & 734 & 0.6 & 0.217 & 656 & 1.0 & 0.217 & 639 \\
\hline
\end{tabular}

TABLE II: Drell-Yan cross section $\sigma\left(p p \rightarrow l^{+} l^{-}\right)$in nmNCSM scenario for the fixed machine energy $\sqrt{s}=7.0 \mathrm{TeV}$. For $K_{Z g g}=0$, the partonic subprocess $g g \rightarrow \gamma, Z \rightarrow l^{+} l^{-}$is absent.

coupling the cross-section $\sigma$ decreases as the $\mathrm{NC}$ scale $\Lambda_{N C}$ increases and finally merges to the SM value at the very high value of $\Lambda_{N C}$. Note that the NCSM contribution to DY process for $K_{Z g g}=0$ almost equal to the $\mathrm{SM}$ value as it receives very small contribution from the quark mediated partonic process and the dominant gluon mediated subprocess is absent due to $K_{Z g g}=0$ (and $K_{\gamma g g}=0$ ). That causes this curve as the lowest (almost) horizontal curve, hence independent of $\Lambda_{N C}$ scale. In Table II we present the leading order cross sections estimated for different $\Lambda_{N C}=0.4,0.6$ and $1.0 \mathrm{TeV}$ corresponding to $K_{Z g g}=0,-0.108$ and 0.217 .

After exploring the additional NC contributions coming towards the Drell-Yan production and how different NC parameters can affect such processes, now we would like to point out some of the very characteristic distributions attributed to noncommutativity. Since space-time noncommutativity essentially breaks the Lorentz invariance which includes the rotational invariance around beam direction, that can contribute to an anisotropic azimuthal distribution. Angular distributions of the final lepton can thus carry this signature on noncommutativity. Similar feature is noted in many different process earlier related with the NC phenomenology, nevertheless we would like to present this distribution in our context. We show the azimuthal angular distribution for the final lepton in Fig. 6. On the left plot this distribution of the azimuthal angle is shown for Drell-Yan events if noncommutative effect is there. While the anisotropic effect is not much visible here under the considerably large crosssection, it would be evident in the right plot where normalized distribution is demonstrated for that same azimuthal angle. This figure is generated corresponding to different scales $\Lambda_{N C}=0.6 \mathrm{TeV}$ and $1 \mathrm{TeV}$. Also, for each $\Lambda_{N C}$, we have selected $K_{Z g g}=-0.108$ and 0.217 , respectively.

From the Fig. 6right plot, we see that the azimuthal distribution of leptons oscillates over 

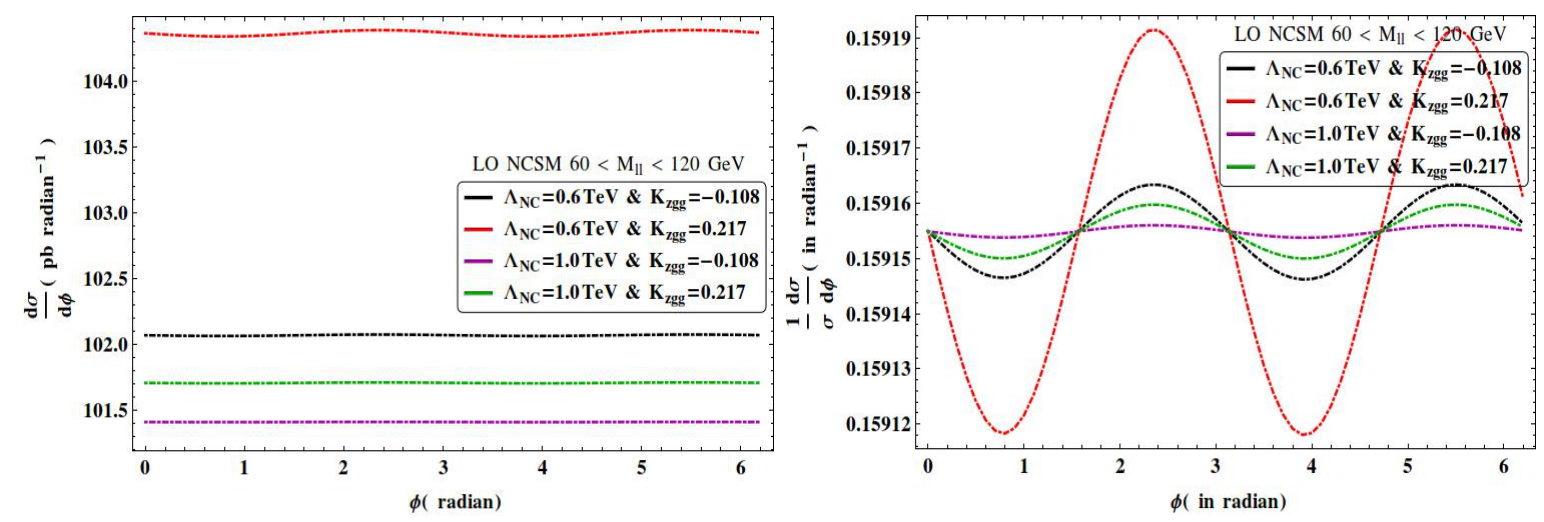

FIG. 6: (Color online). $\frac{d \sigma}{d \phi}$ as a function of $\phi$ for $p p \rightarrow(\gamma, Z) \rightarrow l^{+} l^{-}(l=e, \mu)$ for $\Lambda_{N C}=0.6 \mathrm{TeV}$, $1.0 \mathrm{TeV}$ and $K_{Z g g}=-0.108$ and 0.217 , respectively.

$\phi$, reaching at their maxima at $\phi=2.342 \mathrm{rad}$ and $5.489 \mathrm{rad}$. The two intermediate minimas are located at $\phi=0.783 \mathrm{rad}$ and $3.931 \mathrm{rad}$. Also note that the azimuthal distribution $\frac{d \sigma}{d \phi}$ is completely flat in the SM. A departure from the flat behaviour in the NCSM is due to the term $p_{4} \Theta p_{3}(\sim \cos \theta+\sin \theta(\cos \phi+\sin \phi))$ term in the azimuthal distribution which brings $\phi$ dependence. There still be this feature of azimuthal distribution, even if one deviate from taking the simple form of $\Theta_{\mu \nu}$, however the location of peak positions shift. Such an azimuthal distribution irrespective of peak positions clearly reflects the exclusive nature of spacetime noncommutativity which is rarely to be found in other classes of new physics models and can be tested at LHC.

\section{SUMMARY AND CONCLUSION}

The idea that spacetime can become noncommutative at high energy have drawn attention after the recent advance in string thery. In this paper we have explored the NC effect in the Drell-Yan lepton pair production $p p \rightarrow(\gamma, Z) \rightarrow l^{+} l^{-}$at the Large Hadron Collider. Two new vertices $Z g g$ and $\gamma g g$ (absent in the SM) are being found to play crucial role giving rise new partonic sub-process $g g \stackrel{\gamma, Z}{\longrightarrow} l^{+} l^{-}$(absent in the $\mathrm{SM}$ ). For $\sqrt{s}=7 \mathrm{TeV}$, as the coupling parameter $K_{Z g g}$ (corresponding to the new verices $Z g g$ ) changes from -0.108 to 0.217 , the cross section $\sigma$ increases from $637(660)$ pb to $639(734)$ pb corresponding to $\Lambda_{N C}=1(0.4) \mathrm{TeV}$. The azimuthal distribution $\frac{d \sigma}{d \phi}$, completely $\phi$ independent in the SM, deviates substantially in the NCSM. Thus the noncommutative geometry is quite rich in 
terms of its phenomenological implications, which are worthwhile to explore in the $\mathrm{TeV}$ scale Large Hadron Collider.

\section{Acknowledgments}

The work of P.K.Das is supported in parts by the CSIR Project (Ref. No. 03(1244)12/EMR-II) and the BRNS Project (Ref. No.2011/37P/08/BRNS). The authors

would like to thank Mr. Atanu Guha for useful discussions. Selvaganapathy J would also like to acknowledge his colleague, Mr. Aneesh K.P (recently deceased) for useful discussions.

\section{Appendix A: Feynman rules}

The fermion $f$ (quark $q$ and lepton $l$ ) coupling to photon and $Z$ bosons, to order $\mathcal{O}(\Theta)$ is given by

$$
\begin{gathered}
\gamma f \bar{f}: i e Q_{f}\left\{\gamma_{\mu}+\left[\frac{i}{2}+\left(\frac{P_{o} \Theta P_{i}}{8}\right)\right]\left[\left(P_{o} \Theta\right)_{\mu}\left(\Delta \not P_{i}\right)+\left(\Theta P_{i}\right)_{\mu}\left(\Delta \not P_{o}\right)-\left(P_{o} \Theta P_{i}\right) \gamma_{\mu}\right]\right\}(A 1) \\
Z f \bar{f}: \frac{i e Q_{f}}{\sin \left(2 \theta_{W}\right)}\left\{\gamma_{\mu} \Gamma_{A}^{-}(f)+\left[\frac{i}{2}+\left(\frac{P_{o} \Theta P_{i}}{8}\right)\right]\right. \\
\left.\left[\left(P_{o} \Theta\right)_{\mu}\left(\Delta \not P_{i}\right) \Gamma_{A}^{-}(f)+\left(\Theta P_{i}\right)_{\mu}\left(\Delta \not P_{o}\right) \Gamma_{A}^{-}(f)-\left(P_{o} \Theta P_{i}\right) \gamma_{\mu} \Gamma_{A}^{-}(f)\right]\right\}
\end{gathered}
$$

Here we follow the following notation: $i: i n, o:$ out and $\Delta \not P_{\text {in }, \text { out }}=\mathbb{P}_{\text {in,out }}-m$. Also $\Gamma_{A}^{-}(f)=c_{V}^{f}-c_{A}^{f} \gamma^{5} ; \quad c_{V}^{f}=I_{3}^{f}-2 Q_{f} \sin ^{2}\left(\theta_{W}\right) ; \quad c_{A}^{f}=I_{3}^{f} . Q_{f}$ is the e.m. charge, and $I_{3}^{f}$ is the third component of the week isospin of the fermion $f$ (quark $(q)$ or lepton $(l)$ ). The factors $\theta_{3}$ and $\zeta_{3}$ arises in $\gamma-g-g$ and $Z-g-g$ are given by [21],

$$
\begin{aligned}
\theta_{3}^{\mu \nu \rho}\left(k_{1}, k_{2}, k_{3}\right) & =\theta_{3}\left[\left(\mu, k_{1}\right),\left(\nu, k_{2}\right),\left(\rho, k_{3}\right)\right] \\
& =-\left(k_{1} \Theta k_{2}\right)\left[\left(k_{1}-k_{2}\right)^{\rho} \eta^{\mu \nu}+\left(k_{2}-k_{3}\right)^{\mu} \eta^{\nu \rho}+\left(k_{3}-k_{1}\right)^{\nu} \eta^{\rho \mu}\right] \\
& -\Theta^{\mu \nu}\left[k_{1}^{\rho}\left(k_{2} \cdot k_{3}\right)-k_{2}^{\rho}\left(k_{1} \cdot k_{3}\right)\right]-\Theta^{\nu \rho}\left[k_{2}^{\mu}\left(k_{3} \cdot k_{1}\right)-k_{3}^{\mu}\left(k_{2} \cdot k_{1}\right)\right] \\
& -\Theta^{\rho \mu}\left[k_{3}^{\nu}\left(k_{1} \cdot k_{2}\right)-k_{1}^{\nu}\left(k_{3} \cdot k_{2}\right)\right]+\left(\Theta k_{2}\right)^{\mu}\left[\eta^{\nu \rho} k_{3}^{2}-k_{3}^{\nu} k_{3}^{\rho}\right] \\
& +\left(\Theta k_{3}\right)^{\mu}\left[\eta^{\nu \rho} k_{2}^{2}-k_{2}^{\nu} k_{2}^{\rho}\right]+\left(\Theta k_{3}\right)^{\nu}\left[\eta^{\mu \rho} k_{1}^{2}-k_{1}^{\mu} k_{1}^{\rho}\right]+\left(\Theta k_{1}\right)^{\nu}\left[\eta^{\mu \rho} k_{3}^{2}-k_{3}^{\mu} k_{3}^{\rho}\right] \\
& +\left(\Theta k_{1}\right)^{\rho}\left[\eta^{\mu \nu} k_{2}^{2}-k_{2}^{\mu} k_{2}^{\nu}\right]+\left(\Theta k_{2}\right)^{\rho}\left[\eta^{\mu \nu} k_{1}^{2}-k_{1}^{\mu} k_{1}^{\nu}\right],
\end{aligned}
$$


and

$$
\zeta_{3}=\frac{1}{3 g_{3}^{2}}-\frac{1}{6 g_{4}^{2}}+\frac{1}{6 g_{5}^{2}}
$$

where $g_{3}, g_{4}$ and $g_{5}$ are the moduli parameters defined in [18].

\section{Appendix B: Squared-amplitude terms}

The amplitude-squared terms for the quark-(anti)-quark initiated partonic sub-process $q \bar{q} \rightarrow(\gamma, Z) \rightarrow l^{+} l^{-}$from the Feynman diagram 1(a),

$$
\begin{aligned}
& \overline{\left|\mathcal{M}_{q, \gamma}\right|^{2}}=\left(\frac{A F_{1}}{3}\right)\left[\left(p_{1} \cdot p_{3}\right)\left(p_{2} \cdot p_{4}\right)+\left(p_{1} \cdot p_{4}\right)\left(p_{2} \cdot p_{3}\right)\right] \\
\overline{\left|\mathcal{M}_{q, Z}\right|^{2}} & =\left(\frac{B F_{1}}{3}\right)\left(c_{A}^{l^{2}}+c_{V}^{l^{2}}\right)\left(c_{A}^{q^{2}}+c_{V}^{q^{2}}\right)\left[\left(p_{1} \cdot p_{3}\right)\left(p_{2} \cdot p_{4}\right)+\left(p_{1} \cdot p_{4}\right)\left(p_{2} \cdot p_{3}\right)\right] \\
& +\left(\frac{B F_{1}}{3}\right) c_{A}^{l} c_{V}^{l} c_{A}^{q} c_{V}^{q}\left[\left(p_{1} \cdot p_{4}\right)\left(p_{2} \cdot p_{3}\right)-\left(p_{1} \cdot p_{3}\right)\left(p_{2} \cdot p_{4}\right)\right]
\end{aligned}
$$

and

$$
\begin{aligned}
2 \operatorname{Re} \overline{\left|\mathcal{M}_{q, \gamma}\right|^{\dagger}\left|\mathcal{M}_{q, Z}\right|} & =\left(\frac{2 C F_{1}}{3}\right) c_{A}^{l} c_{A}^{q}\left[\left(p_{1} \cdot p_{3}\right)\left(p_{2} \cdot p_{4}\right)+\left(p_{1} \cdot p_{4}\right)\left(p_{2} \cdot p_{3}\right)\right] \\
& -\left(\frac{2 C F_{1}}{3}\right) c_{V}^{l} c_{V}^{q}\left[\left(p_{1} \cdot p_{4}\right)\left(p_{2} \cdot p_{3}\right)-\left(p_{1} \cdot p_{3}\right)\left(p_{2} \cdot p_{4}\right)\right]
\end{aligned}
$$

Here $A=\frac{128 \pi^{2} \alpha^{2} Q_{l}^{2} Q_{q}^{2}}{\hat{s}^{2}}, \quad B=\frac{128 \pi^{2} \alpha^{2} Q_{l}^{2} Q_{q}^{2}}{\operatorname{Sin}^{4} 2 \theta_{W}\left[\left(\hat{s}-M_{z}^{2}\right)^{2}+\left(M_{Z} \Gamma_{Z}\right)^{2}\right]}, \quad C=\frac{128 \pi^{2} \alpha^{2} Q_{l}^{2} Q_{q}^{2}\left(\hat{s}-M_{z}^{2}\right)}{\hat{s}\left[\left(\hat{s}-M_{z}^{2}\right)^{2}+M_{z}^{2} \Gamma_{z}^{2}\right]}$, $F_{1}=\left[1+\frac{\left(P_{2} \Theta P_{1}\right)^{2}}{4}\right]\left[1+\frac{\left(P_{4} \Theta P_{3}\right)^{2}}{4}\right]$ and $\hat{s}=s x_{1} x_{2}$. Note that ampltude-square terms goes as $\mathcal{O}\left(1, \frac{1}{\Lambda_{N C}^{2}}, \frac{1}{\Lambda_{N C}^{4}}\right)$, respectively.

For the gluon initiated partonic sub-process $g g \rightarrow(\gamma, Z) \rightarrow l^{+} l^{-}$, the squared-amplitude terms from the Feynman diagram 1(b), are given by

$$
\overline{\left|\mathcal{M}_{g, \gamma}\right|^{2}}=4 D F_{2}\left(p_{3 \rho} p_{4 \sigma}+p_{3 \sigma} p_{4 \rho}-\eta_{\rho \sigma} p_{3} \cdot p_{4}\right) \cdot\left(\eta_{\nu \beta}{\overline{\theta_{3}}}^{\alpha \beta \sigma} \eta_{\alpha \mu}{\overline{\theta_{3}}}^{\mu \nu \rho}\right)
$$

Similarly,

$$
\begin{gathered}
\overline{\left|\mathcal{M}_{g, Z}\right|^{2}}=4 G F_{2}\left(c_{A}^{l^{2}}+c_{V}^{l^{2}}\right)\left(p_{3 \rho} p_{4 \sigma}+p_{3 \sigma} p_{4 \rho}-\eta_{\rho \sigma} p_{3} \cdot p_{4}\right)\left(\eta_{\nu \beta}{\overline{\theta_{3}}}^{\alpha \beta \sigma} \eta_{\alpha \mu}{\overline{\theta_{3}}}^{\mu \nu \rho}\right) \\
2 R \overline{\overline{\left|\mathcal{M}_{g, \gamma} \mathcal{M}_{z}^{\dagger}\right|}}=4 H F_{2} c_{V}^{l}\left(p_{3 \rho} p_{4 \sigma}+p_{3 \sigma} p_{4 \rho}-\eta_{\rho \sigma} p_{3} \cdot p_{4}\right) \cdot\left(\eta_{\nu \beta}{\overline{\theta_{3}}}^{\alpha \beta \sigma} \eta_{\alpha \mu}{\overline{\theta_{3}}}^{\mu \nu \rho}\right)
\end{gathered}
$$


Here $F_{2}=\left[1+\frac{\left(P_{4} \Theta P_{3}\right)^{2}}{4}\right], D=2\left(\frac{\pi \alpha \sin \left(2 \theta_{w}\right) K_{\gamma g g}}{\hat{s}}\right)^{2}, G=\left[\frac{2 \pi^{2} \alpha^{2} k_{z g g}^{2}}{\left[\left(\hat{s}-M_{z}^{2}\right)^{2}+M_{z}^{2} \Gamma_{z}^{2}\right]}\right]$,

$H=\sin \left(2 \theta_{w}\right) K_{\gamma g g} K_{z g g}\left[\frac{4 \pi^{2} \alpha^{2}}{\hat{s}}\left(\frac{\hat{s}-M_{z}^{2}}{\left[\left(\hat{s}-M_{z}^{2}\right)^{2}+M_{z}^{2} \Gamma_{z}^{2}\right]}\right)\right]$.

The quantity $\bar{\theta}_{3}$ appearing in several squared-amplitude terms, is given by

$$
\begin{aligned}
\bar{\theta}_{3}^{\mu \nu \rho} & =-\left(p_{1} \Theta p_{2}\right)\left[\left(p_{1}-p_{2}\right)^{\rho} \eta^{\mu \nu}+2\left(p_{2}^{\mu} \eta^{\nu \rho}-p_{1}^{\nu} \eta^{\rho \mu}\right)\right] \\
& +\left(p_{1} \cdot p_{2}\right)\left[\Theta^{\mu \nu}\left(p_{1}-p_{2}\right)^{\rho}-2\left(\left(p_{2} \Theta\right)^{\mu} \eta^{\nu \rho}+\left(p_{1} \Theta\right)^{\nu} \eta^{\mu \rho}\right)\right]+\left[\left(p_{2} \Theta\right)^{\mu} p_{1}^{\nu}+\left(p_{1} \Theta\right)^{\nu} p_{2}^{\mu}\right]\left(p_{1}+p_{2}\right)^{\rho}
\end{aligned}
$$

Note that ampltude-square terms goes as $\mathcal{O}\left(\frac{1}{\Lambda_{N C}^{4}}, \frac{1}{\Lambda_{N C}^{8}}\right)$, respectively.

In evaluating the matrix element square, we have used the following orthonormality condition

$$
\begin{aligned}
& \sum_{\lambda, \lambda^{\prime}} \epsilon_{\mu^{\prime}}^{* a^{\prime}}\left(p_{1}, \lambda_{1}^{\prime}\right) \epsilon_{\mu}^{a}\left(p_{1}, \lambda_{1}\right)=-\eta_{\mu^{\prime} \mu} \delta_{a^{\prime} a} \\
& \sum_{\lambda, \lambda^{\prime}} \epsilon_{\nu}^{b}\left(p_{2}, \lambda_{2}\right) \epsilon_{\nu^{\prime}}^{* b^{\prime}}\left(p_{2}, \lambda_{2}^{\prime}\right)=-\eta_{\nu^{\prime} \nu} \delta_{b^{\prime} b}
\end{aligned}
$$

and the color algebra $\sum_{a a^{\prime} b b^{\prime}} \delta_{b b^{\prime}} \delta_{a a^{\prime}} \delta^{a b} \delta_{a^{\prime} b^{\prime}}=\sum_{a b} \delta^{a b} \delta_{a b}=\sum_{a=1}^{8} \delta_{a a}=8$

\section{Appendix C: Antisymmetric tensor $\Theta_{\mu \nu}$ and $\Theta$ wieghted dot product}

The antisymmetric tensor $\Theta_{\mu \nu}=\frac{1}{\Lambda_{N C}^{2}} c_{\mu \nu}$ has 6 independent components corresponding to $c_{\mu \nu}=\left(c_{o i}, c_{i j}\right)$ with $i, j=1,2,3$. Assuming them as the non-vanishing components, we can write them as follows:

$$
c_{o i}=\xi_{i}, \quad c_{i j}=\epsilon_{i j k} \chi^{k}
$$

The NC antisymmetric tensor $\Theta_{\mu \nu}$ is analogous to the electro-magnetic(e.m.) field strength tensor $F_{\mu \nu}: \xi_{i}$ and $\chi_{i}$ are like the electric and magnetic field vectors. Setting $\xi_{i}=(\vec{E})_{i}=\frac{1}{\sqrt{3}}$ and $\chi_{i}=(\vec{B})_{i}=\frac{1}{\sqrt{3}}$ with $i=1,2,3$ and noting the fact that $\xi_{i}=-\xi^{i}, \chi_{i}=-\chi^{i}$, the normalization condition $\xi_{i} \xi^{j}=\frac{1}{3} \delta_{i}^{j}$ and $\chi_{i} \chi^{j}=\frac{1}{3} \delta_{i}^{j}$, we may write $\Theta_{\mu \nu}$ as

$$
\Theta_{\mu \nu}=\frac{1}{\sqrt{3} \Lambda_{N C}^{2}}\left(\begin{array}{cccc}
0 & 1 & 1 & 1 \\
-1 & 0 & -1 & 1 \\
-1 & 1 & 0 & -1 \\
-1 & -1 & 1 & 0
\end{array}\right)
$$


Using these we may write the $\Theta$-weighted dot-product as follows:

$$
\begin{aligned}
& p_{2} \Theta p_{1}=\frac{\hat{s}}{2 \sqrt{3} \Lambda_{N C}^{2}} \\
& p_{4} \Theta p_{3}=\frac{\hat{s}}{2 \sqrt{3} \Lambda_{N C}^{2}}[\cos \theta+\sin \theta(\cos \phi+\sin \phi)]
\end{aligned}
$$

Here we have not considered the effect of earth rotation on the anti-symmetric tensor $\Theta_{\mu \nu}$ in DY process. This will be reported elsewhere [26].

[1] CMS Collaboration, S. Chatrchyan et al., Observation of a new boson at a mass of $125 \mathrm{GeV}$ with the CMS experiment at the LHC, Phys.Lett. B716 (2012) 30-61,

[2] ATLAS Collaboration, G. Aad et al., Observation of a new particle in the search for the Standard Model Higgs boson with the ATLAS detector at the LHC, Phys.Lett. B716 (2012) $1-29$.

[3] H. S. Snyder, The E.M. field in quantized space-time, Phys. Rev. D71 (1947) 38.

[4] N. Seiberg and E. Witten, String Theory and Noncommutative Geometry, Journal of High Energy Phys 09 (1999) 032.

[5] The OPAL collaboration, Test of non-commutative QED in the process $e^{+} e^{-} \rightarrow \gamma \gamma$ at LEP, Phys. Lett. B568 (2003) 181.

[6] I. F. Riad and M. M. Sheikh-Jabbari, On Moyal-Weyl map, Journal of High Energy Phys 08 (2000) 45.

[7] B. Jurco and P. Schupp, Noncommutative Yang-Mills from equivalence of star products, Eur. Phys. Journal C14 (2000) 367.

[8] J. M. Gracia-Bondia and J. C.Varilly, QED in external fields from the spin representation, J.Math.Phys. 35 (1904) 3340.

[9] J. Hewett, F. J. Petriello and T. G. Rizzo, in Signals for Noncommutative QEC at High Energy $e^{+} e^{-}$colliders, eConf C010630, E3064 (2001).

[10] J. Hewett et al. , Signals for noncommutative interactions at linear colliders Phys. Rev. D64 (2001) 075012.

[11] P. Mathews, Compton Scattering in Noncommutative. Space-Time at the NLC Phys. Rev. D63 (2001) 075007.

[12] T. G. Rizzo, QED at future e+ e-colliders Int. Jour. of Mod. Phys A18 (2003) 2797. 
[13] X. Calmet, B. Jurco, P. Schupp, J. Wess and M. Wohlgenannt, The standard model on noncommutative spacetime, Eur. Phys. Journal C23 (2002) 363.

[14] X. Calmet and M. Wohlgenannt, Effective field theories on noncommutative space-time, Phys. Rev. D68 (2003) 025016.

[15] A. Prakash, A. Mitra and P. K. Das, $e^{-} e^{+} \rightarrow \mu^{-} \mu^{+}$scattering in the NCSM, Phys. Rev. D82 (2010) 055020.

[16] A. Prakash and P. K. Das, Laboratory Frame Analysis of $e^{-} e^{+} \rightarrow \mu^{-} \mu^{+}$scattering in the Noncommutative Standard Model, Int.J.Mod.Phys. A27 (2012) 1250141.

[17] Ravi. S. Manohar, Selvaganapathy J and P. K. Das, Probing space-time noncommutativity in the top quark pair production at e+e-collider, Int.J.Mod.Phys. A29 (2014) 1450156.

[18] W. Behr, N. G. Deshpande, G. Duplancic,P. Schupp, J. Trampetic and J. Wess, The $Z \rightarrow \gamma \gamma$, gg Decays in the Noncommutative Standard Model, Eur.Phys.J C29 (2003) 441.

[19] Selvaganapathy. J, Prasanta Kr. Das and Partha Konar, Search for associated production of Higgs with $Z$ boson in the noncommutative Standard Model at linear colliders, Int. J. Mod. Phys. A30 (2015) 155015.

[20] B. Melić, K. P. Kumericki, J. Trampetic, P. Schupp and M. Wohlgenannt, The standard model on non-commutative space-time: Electroweak currents and Higgs sector, Eur. Phys. Journal C42 (2005) 483.

[21] B. Melić et al. , The standard model on non-commutative space-time: Strong interaction included, Eur. Phys. Journal C42 (2005) 499.

[22] I. Hinchliffe, N. Kersting and Y. L. Ma, Review of the Phenomenology of Noncommutative Geometry, International Journal of Modern Physics, A19 (2004) 179.

[23] Ana Alboteanu, Thorsten Ohl and Reinhold Rückl, Probing the Noncommutative Standard Model at Hadron Colliders, Phys. Rev. D74 (2006) 096004.

[24] T. Ohl and C. Speckner, The Noncommutative Standard Model and Polarization in Charged Gauge Boson Production at the LHC, Phys. Rev. D82 (2010) 116011.

[25] S. Y. Ayazi, S. Esmaeili and M. M. Najafabadu, Single top quark production in t-channel at the LHC in Noncommutative Space-Time Phys. Lett. B712 (2012) 93.

[26] Selvaganapathy. J, Atanu Guha, Partha Konar and Prasanta Kr. Das, Work is in progress.

[27] CMS Collaboration, Measurement of the Drell-Yan Cross Section in pp Collisions at $\sqrt{s}=$ 7 TeV, JHEP 10 (2011) 007; arXiv:1108.0566 [hep-ex] 